\title{
Creativity East and West: Perspectives and Parallels
}

\section{东方和西方的创造力研究：观点及相似性}

\author{
Michael W. Morris and Kwok Leung (梁觉)
}

摘要

本期编辑论坛一“东方和西方的创造力研究”一包括有关创造力的文化差异问题的 五篇不同研究视角的文章以及两篇整合性的评论。这些文章的研究文献包括历史测量 法、实验法以及组织研究, 它们采用国别间的比较和启动操纵的方式探讨了文化的影 响, 还考查了人们在如何创造性地工作以及怎样评估创造力方面表现出的文化模式。 这个导言指出, 这些不同视角的研究发现具有可比之处, 表明创造力研究中既存在文 化上的普遍性, 也存在系统的差异。许多差异可以用创造力意味着既新颖又实用的解 决方案这一模型来解释, 不过西方的社会规范优先强调新䓉性而东方的规范则将实用 性至于优先位置。两篇评论根据创新发生的过程一在微观的认知层次和宏观的社会层 次_上一的观点对于上述论点作了详尽的说明。

关键词：创造力, 文化差异, 东亚, 创新, 社会规范

\section{Greativity in Eastern and Western Givilizations: The Lessons of Historiometry}

\section{东方及西方文明中的创造力：历史测量学的教训}

\section{Dean Keith Simonton (赛门顿) and Shing-Shiang Ting (丁兴详)}

摘要

促进具有高度影响的创造力的基本因素是什么? 这些因素在远东与西方文明中有何不 同? 许多研究者采用历史测留学探讨了这些问题。历史测量学是通过对历史及传记资 料进行客观和量化的分析, 来对人类行为进行通则式假设检验的一种方法。这些研究 要么采用聚合层次的分析 (如：创造性活动的世代性时间序列分析)，要么采用个体 层次的分析 (如：创造性成就的横截式研究)。而且, 采用这两种方法获得的实证发 现可以归为两个类别的东西方比较：(a) 共享的变量与趋同的结果, 与 (b) 共享的变 $1 \mathrm{I}$ 与发散的结果。回顾了这两大类别中具有代表性的研究发现之后, 我们讨论了这些结 果对于东西方文明中具有高度影响力的创造力本质所包含的意义, 并探讨了未来历史 测情学可能探讨的研究领域。

关键词：文明，创造力, 普遍化, 历史测备学, 时代精神 


\title{
Creativity: The Influence of Cultural, Social, and Work Contexts
}

\section{创造力：文化、社会及工作情境的影响}

\section{Miriam Erez and Rikki Nouri}

摘要

本文旨在回答创造力究竟是普遍的还是具有文化特殊性的这个问题。我们建立了一个 概念框架, 以两种方式来扩展现有的知识。第一, 它区分了创造力的两个维度 : 新颖 性和有用性以及它们和文化之间的关系。第二, 它澄清了社会情境是怎样调节文化和 创造力之间的关系。相比在私人工作情境下, 没有别人观察个人是以规范的方式还是 独特的方式来工作, 我们集中关注由于他人在场而导致的文化差异比较显都的社会情 境。此外, 我们还提出, 任务结构, 即任务的结构是严密的还是松散的, 是一个重要 的调节文化和创造力之间关系的情境因素。最后, 我们提供了几个指引未来研究方问 的主张。

关键词：创造力，文化，新䓉性，社会情境，任务情境，有用性

\section{Asian-Americans' Greative Styles in Asian and American Situations: Assimilative and Contrastive Responses as a Function of Bicultural Identity Integration}

\section{亚洲和美国情境中的亚裔美国人的创造性风格： 双文化身份整合导致的同化和对比反应}

\author{
Aurelia Mok and Michael W. Morris
}

摘要

双文化的个体在两种文化身份的整合程度上有所不同。例如, 在亚裔美国人中，一些 人觉得自己的亚洲和美国经历是相吻合的，而另一些人则觉得两种经历是冲突的。以 往关于判断的研究发现, 这种个别差异会影响双文化个体对于引发他们文化的特定情 境做出的反应。具有高双文化身份整合 (BII) 的亚裔美国人会同化到被引发的文化规 范中（例如, 当处在引发美国文化的情境中, 他们通常表现出美国式的判断)，而低 双文化整合的亚裔美国人则表现出与引发线索相反的判断（例如，他们在美国情境下 通常表现出亚洲式的判断）。我们探讨了这种动态性是否也同样会影响创造性的业 绩, 即在美国规范比东亚规范更鼓励新颖性方面, 是否存在跨文化的差异。我们通过 两个实验发现, 引发美国 (对比亚洲) 文化的线索增加了高BII的亚裔美国人在解决发 散思维任务中表现出的新颖性 (同化反应) , 但却降低了低BII的亚裔美国人在上述任 务中的新渘性 (对比反应) 。我们讨论了这些发现对于文化和创造力研究的理论意 义, 以及对于企业寻求促进创造力所具有的现实意义。

关键词：双文化身份，创造力，文化规范

(C) 2010 The Intcrnational Association for Chincsc Management Research 


\title{
A Missing Piece of the Puzzle: The Organizational Context in Cultural Patterns of Creativity 缺失的片段：何造力的文化模式中的组织情境
}

\author{
Jing Zhou (周京) and Yanjie Su (苏彦捷)
}

摘要

本文首先回顾了采用实验室任务和测验对创造力进行的跨文化研究, 特别关注了东西 方文化下的相似性和差异性。在此基础上, 我们提出在未来开展有关工作环境中的创 造力的跨文化研究的若干方向。我们强调，需要来解释文化差异导致创造力差异的原 因; 而且研究应直接考察情境因素对创造力的影响, 而不只是停留在假定的层面。为 此, 将采用实验室任务的跨文化研究和探讨员工创造力的组织研究这两个方面的文献 进行整合, 会使研究者受益匪浅。我们也呼吁开展更多的实证研究, 探讨文化对工作 场合中的创造力的影响, 在中国更需要这样的实证研究。

关键词：同事的影响，创造力，跨文化，创新、领导，社会网络

\section{Culture and the Assessment of Creativity 文化与创造力评估}

\section{Paul S. Hempel and Christina Sue-Chan}

摘要

在文化与创造力的实验研究中, 通常会对想法进行不同的测量; 而在组织情境中的创 造力研究吅, 则会明确地定义何谓创造力, 并邀请某领域的专家作为评价者来估计文 化对于创造力评估的影响。然而, 来自不同的创造性活动 (领域) 的学科专家们, 在 评估一个创造性想法时所考虑的究竟是哪些具体特征, 我们对其知之甚少。在本文 中1, 我们根据访谈所搜集到的原始资料, 对文化对评估整体创造力的两个常网维度 (即, 新颖性和有用性) 的影响进行了回顾、概念性的分析和说明。以外派人员为背 景, 我们提出了一个研究框架和若干命题, 整合了文化经验、创造力标准和评估者对 创造力评估的看法。最后, 我们讨论了通过关注创造力评估背后的过程来推动文化和 创造力研究的途径。

关键词：创造力评估，中国，创造力 


\section{Human Creativity: Reflections on the Role of Culture}

\section{人类的创造力：对文化作用的反省}

\section{Carsten K.W. De Dreu}

摘要

对于本期编辑论坛的文章进行的评论, 将讨论三个问题：（1）我们应当如何对于创造 性成果进行操作化并进行测量，从而能够对于创造力的文化差异进行合理的分析?

（2）文化是否不仅影响原创性和实用性的价值而且还影响原创且有用的想法和洞见实 现的过程? 和 (3) 文化是否会影响个体被激励从事创造性的工作? 以近期关于创造力 的工作作为起点, 并根据本编辑论坛中报告的主要发现, 我提出, 将创造性产品与创 造性过程分开, 将有利于文化和创造力的新近研究。我还认为, 思考个体和群体何时 以及怎样获得创造力, 以及他们为什么将具有创造力看得很重要, 将能够更好地理解 文化的本质和功能。

关键词：创造力，文化，二元过程模型，创新

\section{Culture and Greativity: A Process Model 文化与创造力：一个过程模型}

\section{Chi-yue Chiu (赵志裕) and Letty Y-Y. Kwan}

摘要

在这个论坛上发表的文章, 对文化在创造力中的作用提出了很多创见。在创造力领域 中, 有两个常见的主题：(1) 创新的起源, 以及 (2) 哪些概念上的改良能推动这个 领域的发展。在这篇评论中, 我们首先讨论这些文章在这两个主题上的贡献。然后, 我们提出一个创造力的过程模型, 并解释文化在每个知识创造阶段中的作用。我们认 为，成功的创新涉及以下三个阶段中的一个或多个：（1）缔造新想法；（2）选择、 编辑和推销新想法; 和 (3) 让新想法被市场接受。各个阶段的理想成果有所不同, 但 其过程都受到文化的影响。具体而言, 现有的文化知识是评估新想法的原创性的标 准; 对文化共识的设想为选择、编辑和推销想法提供了规范性的基础; 而现实的文化 规范则能决定一个想法在多大程度上被文化所接纳。更进一步, 不同的社会和心理过 程在创造力过程的不同阶段都起作用, 而文化则通过影响这些社会和心理的过程来影 响创造力过程的结果。

关键词：知识, 创造力, 文化, 多元文化经验 